\title{
Erratum to: A Donsker Delta Functional Approach to Optimal Insider Control and Applications to Finance
}

\author{
Olfa Draouil $^{1}$ • Bernt $\emptyset \mathrm{ksendal}^{2,3}$
}

Published online: 24 November 2015

(C) School of Mathematical Sciences, University of Science and Technology of China and Springer-Verlag Berlin Heidelberg 2015

\section{Erratum to: Commun. Math. Stat. (2015) 3:365-421 DOI 10.1007/s40304-015-0065-y}

Unfortunately, in the original publication of the article some equations appeared incorrectly. The corrected equations are given below:

- In (1.1) it should be added that the filtration $\mathbb{H}$ is assumed to be right-continuous, i.e., that

$$
\mathcal{H}_{t}=\mathcal{H}_{t^{+}}=\bigcap_{s>t} \mathcal{H}_{s} .
$$

- The third line of (1.4) should read

$$
\gamma(t, x, u, y, \zeta)=\gamma(t, x, u, y, \zeta, \omega):\left[0, T_{0}\right] \times \mathbb{R} \times \mathbb{R} \times \mathbb{R} \times \mathbb{R} \times \Omega \mapsto \mathbb{R}
$$

- In (2.5), which reads

$$
Y=Y\left(T_{0}\right) ; \text { where } Y(t)
$$

The online version of the original article can be found under doi:10.1007/s40304-015-0065-y.

\footnotetext{
$\bowtie \quad$ Bernt Øksendal

oksendal@math.uio.no

Olfa Draouil

olfadraouil@hotmail.fr

1 Department of Mathematics, University of Tunis El Manar, Tunis, Tunisia

2 Department of Mathematics, University of Oslo, P.O. Box 1053, Blindern, 0316 Oslo, Norway

3 Norwegian School of Economics (NHH), Helleveien 30, 5045 Bergen, Norway
} 


$$
=\int_{0}^{t} \beta(s) \mathrm{d} B(s)+\int_{0}^{t} \int_{\mathbb{R}} \psi(s, \zeta) \tilde{N}(\mathrm{~d} s, \mathrm{~d} \zeta) \text {, for } t \in\left[0, T_{0}\right],
$$

we should add the assumption that $\beta \neq 0$.

- In the formulas (2.7)-(2.14) and in (6.107), (6.109), we should replace $\beta$ by $i \beta$, where $i=\sqrt{-1}$.

When this is done, the correct version of (2.7) becomes

$$
\begin{aligned}
\delta_{Y}(y)= & \frac{1}{2 \pi} \int_{\mathbb{R}} \exp ^{\diamond}\left[\int_{0}^{T_{0}} \int_{\mathbb{R}}\left(\mathrm{e}^{i x \psi(s, \zeta)}-1\right) \tilde{N}(\mathrm{~d} s, \mathrm{~d} \zeta)+\int_{0}^{T_{0}} i x \beta(s) \mathrm{d} B(s)\right. \\
& \left.+\int_{0}^{T_{0}}\left\{\int_{\mathbb{R}}\left(\mathrm{e}^{i x \psi(s, \zeta)}-1-i x \psi(s, \zeta)\right) v(\mathrm{~d} \zeta)-\frac{1}{2} x^{2} \beta^{2}(s)\right\} \mathrm{d} s-i x y\right] \mathrm{d} x,
\end{aligned}
$$

and similarly, the correct versions of (2.8)-(2.14) become as follows:

$$
\begin{aligned}
& \mathbb{E}\left[\delta_{Y}(y) \mid \mathcal{F}_{t}\right] \\
& =\frac{1}{2 \pi} \int_{\mathbb{R}} \mathbb{E}\left[\operatorname { e x p } ^ { \diamond } \left[\int_{0}^{T_{0}} \int_{\mathbb{R}}\left(\mathrm{e}^{i x \psi(s, \zeta)}-1\right) \tilde{N}(\mathrm{~d} s, \mathrm{~d} \zeta)+\int_{0}^{T_{0}} i x \beta(s) \mathrm{d} B(s)\right.\right. \\
& \left.\left.+\int_{0}^{T_{0}}\left\{\int_{\mathbb{R}}\left(\mathrm{e}^{i x \psi(s, \zeta)}-1-i x \psi(s, \zeta)\right) v(\mathrm{~d} \zeta)-\frac{1}{2} x^{2} \beta^{2}(s)\right\} \mathrm{d} s-i x y\right] \mid \mathcal{F}_{t}\right] \mathrm{d} x \\
& =\frac{1}{2 \pi} \int_{\mathbb{R}} \exp ^{\diamond}\left[\mathbb { E } \left[\int_{0}^{T_{0}} \int_{\mathbb{R}}\left(\mathrm{e}^{i x \psi(s, \zeta)}-1\right) \tilde{N}(\mathrm{~d} s, \mathrm{~d} \zeta)+\int_{0}^{T_{0}} i x \beta(s) \mathrm{d} B(s)\right.\right. \\
& \left.\left.+\int_{0}^{T_{0}}\left\{\int_{\mathbb{R}}\left(\mathrm{e}^{i x \psi(s, \zeta)}-1-i x \psi(s, \zeta)\right) v(\mathrm{~d} \zeta)-\frac{1}{2} x^{2} \beta^{2}(s)\right\} \mathrm{d} s-i x y \mid \mathcal{F}_{t}\right]\right] \mathrm{d} x \\
& =\frac{1}{2 \pi} \int_{\mathbb{R}} \exp ^{\diamond}\left[\int_{0}^{t} \int_{\mathbb{R}}\left(\mathrm{e}^{i x \psi(s, \zeta)}-1\right) \tilde{N}(\mathrm{~d} s, \mathrm{~d} \zeta)+\int_{0}^{t} i x \beta(s) \mathrm{d} B(s)\right. \\
& \left.+\int_{0}^{T_{0}}\left\{\int_{\mathbb{R}}\left(\mathrm{e}^{i x \psi(s, \zeta)}-1-i x \psi(s, \zeta)\right) v(\mathrm{~d} \zeta)-\frac{1}{2} x^{2} \beta^{2}(s)\right\} \mathrm{d} s-i x y\right] \mathrm{d} x \\
& =\frac{1}{2 \pi} \int_{\mathbb{R}}\left\{\exp ^{\diamond}\left[\int_{0}^{t} \int_{\mathbb{R}}\left(\mathrm{e}^{i x \psi(s, \zeta)}-1\right) \tilde{N}(\mathrm{~d} s, \mathrm{~d} \zeta)\right]\right\} \diamond\left\{\exp ^{\diamond}\left[\int_{0}^{t} i x \beta(s) \mathrm{d} B(s)\right]\right\} \\
& \diamond\left\{\exp ^{\diamond}\left[\int_{0}^{T_{0}}\left\{\int_{\mathbb{R}}\left(\mathrm{e}^{i x \psi(s, \zeta)}-1-i x \psi(s, \zeta)\right) v(\mathrm{~d} \zeta)-\frac{1}{2} x^{2} \beta^{2}(s)\right\} \mathrm{d} s-i x y\right]\right\} \mathrm{d} x \\
& =\frac{1}{2 \pi} \int_{\mathbb{R}} \exp \left[\int_{0}^{t} \int_{\mathbb{R}} i x \psi(s, \zeta) \tilde{N}(\mathrm{~d} s, \mathrm{~d} \zeta)+\int_{0}^{t} i x \beta(s) \mathrm{d} B(s)\right. \\
& \left.+\int_{t}^{T_{0}} \int_{\mathbb{R}}\left(\mathrm{e}^{i x \psi(s, \zeta)}-1-i x \psi(s, \zeta)\right) v(\mathrm{~d} \zeta) \mathrm{d} s-\int_{t}^{T_{0}} \frac{1}{2} x^{2} \beta^{2}(s) \mathrm{d} s-i x y\right] \mathrm{d} x
\end{aligned}
$$


Here we have used that (see e.g. [11, Lemma 3.1])

$$
\exp ^{\diamond}\left[\int_{0}^{T_{0}} i x \beta(s) \mathrm{d} B(s)\right]=\exp \left[\int_{0}^{T_{0}} i x \beta(s) \mathrm{d} B(s)+\frac{1}{2} \int_{0}^{T_{0}} x^{2} \beta^{2}(s) \mathrm{d} s\right]
$$

and

$$
\begin{aligned}
& \exp ^{\diamond}\left[\int_{0}^{T_{0}} \int_{\mathbb{R}}\left(\mathrm{e}^{i x \psi(s, \zeta)}-1\right) \tilde{N}(\mathrm{~d} s, \mathrm{~d} \zeta)\right] \\
& \quad=\exp \left[\int_{0}^{T_{0}} \int_{\mathbb{R}} i x \psi(s, \zeta) \tilde{N}(\mathrm{~d} s, \mathrm{~d} \zeta)-\int_{0}^{T_{0}} \int_{\mathbb{R}}\left(\mathrm{e}^{i x \psi(s, \zeta)}-1-i x \psi(s, \zeta)\right) v(\mathrm{~d} \zeta)\right]
\end{aligned}
$$

We proceed to find

$$
\mathbb{E}\left[D_{t, z} \delta_{Y}(y) \mid \mathcal{F}_{t}\right]
$$

where $D_{t, \zeta}$ denotes the Hida-Malliavin derivative at $(t, \zeta) \in[0, T] \times \mathbb{R}$ with respect to the Poisson random measure $N$ :

First, note that

$$
\begin{aligned}
D_{t, z} \delta_{Y}(y)= & \frac{1}{2 \pi} \int_{\mathbb{R}} D_{t, z} \exp ^{\diamond}\left[\int_{0}^{T_{0}} \int_{\mathbb{R}}\left(\mathrm{e}^{i x \psi(s, \zeta)}-1\right) \tilde{N}(\mathrm{~d} s, \mathrm{~d} \zeta)+\int_{0}^{T_{0}} i x \beta(s) \mathrm{d} B(s)\right. \\
& \left.+\int_{0}^{T_{0}}\left\{\int_{\mathbb{R}}\left(\mathrm{e}^{i x \psi(s, \zeta)}-1-i x \psi(s, \zeta)\right) v(\mathrm{~d} \zeta)-\frac{1}{2} x^{2} \beta^{2}(s)\right\} \mathrm{d} s-i x y\right] \mathrm{d} x \\
= & \frac{1}{2 \pi} \int_{\mathbb{R}} \exp ^{\diamond}\left[\int_{0}^{T_{0}} \int_{\mathbb{R}}\left(\mathrm{e}^{i x \psi(s, \zeta)}-1\right) \tilde{N}(\mathrm{~d} s, \mathrm{~d} \zeta)+\int_{0}^{T_{0}} i x \beta(s) \mathrm{d} B(s)\right. \\
& \left.+\int_{0}^{T_{0}}\left\{\int_{\mathbb{R}}\left(\mathrm{e}^{i x \psi(s, \zeta)}-1-i x \psi(s, \zeta)\right) v(\mathrm{~d} \zeta)-\frac{1}{2} x^{2} \beta^{2}(s)\right\} \mathrm{d} s-i x y\right] \\
& \diamond D_{t, z}\left[\int_{0}^{T_{0}} \int_{\mathbb{R}}\left(\mathrm{e}^{i x \psi(s, \zeta)}-1\right) \tilde{N}(\mathrm{~d} s, \mathrm{~d} \zeta)+\int_{0}^{T_{0}} i x \beta(s) \mathrm{d} B(s)\right. \\
& \left.+\int_{0}^{T_{0}}\left\{\int_{\mathbb{R}}\left(\mathrm{e}^{i x \psi(s, \zeta)}-1-i x \psi(s, \zeta)\right) v(\mathrm{~d} \zeta)-\frac{1}{2} x^{2} \beta^{2}(s)\right\} \mathrm{d} s-i x y\right] \mathrm{d} x \\
= & \frac{1}{2 \pi} \int_{\mathbb{R}} \exp ^{\diamond}\left[\int_{0}^{T_{0}} \int_{\mathbb{R}}\left(\mathrm{e}^{i x \psi(s, \zeta)}-1\right) \tilde{N}(\mathrm{~d} s, \mathrm{~d} \zeta)+\int_{0}^{T_{0}} i x \beta(s) \mathrm{d} B(s)\right. \\
& \left.+\int_{0}^{T_{0}}\left\{\int_{\mathbb{R}}\left(\mathrm{e}^{i x \psi(s, \zeta)}-1-i x \psi(s, \zeta)\right) v(\mathrm{~d} \zeta)-\frac{1}{2} x^{2} \beta^{2}(s)\right\} \mathrm{d} s-i x y\right] \\
& \times\left(\mathrm{e}^{i x \psi(t, z)}-1\right) \mathrm{d} x .
\end{aligned}
$$


Here we have used that

$$
D_{t, z} \int_{0}^{T} \beta(s) \mathrm{d} B(s)=0,
$$

which follows from our assumption that $B$ and $\tilde{N}$ are independent, so for $D_{t, z}$ the random variable $B(s)$ is like a constant.

Using Eq. (2.11) and the Wick chain rule we get

$$
\begin{aligned}
\mathbb{E}[ & \left.D_{t, z} \delta_{Y}(y) \mid \mathcal{F}_{t}\right] \\
= & \frac{1}{2 \pi} \int_{\mathbb{R}} \mathbb{E}\left[\operatorname { e x p } ^ { \diamond } \left[\int_{0}^{T_{0}} \int_{\mathbb{R}}\left(\mathrm{e}^{i x \psi(s, \zeta)}-1\right) \tilde{N}(\mathrm{~d} s, \mathrm{~d} \zeta)+\int_{0}^{T_{0}} i x \beta(s) \mathrm{d} B(s)\right.\right. \\
& \left.+\int_{0}^{T_{0}}\left\{\int_{\mathbb{R}}\left(\mathrm{e}^{i x \psi(s, \zeta)}-1-i x \psi(s, \zeta)\right) v(\mathrm{~d} \zeta)-\frac{1}{2} x^{2} \beta^{2}(s)\right\} \mathrm{d} s-i x y\right] \\
& \left.\times\left(\mathrm{e}^{i x \psi(t, z)}-1\right) \mathrm{d} x \mid \mathcal{F}_{t}\right] \mathrm{d} x \\
= & \frac{1}{2 \pi} \int_{\mathbb{R}} \mathbb{E}\left[\operatorname { e x p } ^ { \diamond } \left[\int_{0}^{T_{0}} \int_{\mathbb{R}}\left(\mathrm{e}^{i x \psi(s, \zeta)}-1\right) \tilde{N}(\mathrm{~d} s, \mathrm{~d} \zeta)+\int_{0}^{T_{0}} i x \beta(s) \mathrm{d} B(s)\right.\right. \\
& \left.\left.+\int_{0}^{T_{0}}\left\{\int_{\mathbb{R}}\left(\mathrm{e}^{i x \psi(s, \zeta)}-1-i x \psi(s, \zeta)\right) v(\mathrm{~d} \zeta)-\frac{1}{2} x^{2} \beta^{2}(s)\right\} \mathrm{d} s-i x y\right] \mid \mathcal{F}_{t}\right] \\
& \times\left(\mathrm{e}^{i x \psi(t, z)}-1\right) \mathrm{d} x \\
= & \frac{1}{2 \pi} \int_{\mathbb{R}} \exp \left[\int_{0}^{t} \int_{\mathbb{R}} i x \psi(s, \zeta) \tilde{N}(\mathrm{~d} s, \mathrm{~d} \zeta)+\int_{0}^{t} i x \beta(s) \mathrm{d} B(s)\right. \\
& \left.+\int_{t}^{T_{0}} \int_{\mathbb{R}}\left(\mathrm{e}^{i x \psi(s, \zeta)}-1-i x \psi(s, \zeta)\right) v(\mathrm{~d} \zeta) \mathrm{d} s-\int_{t}^{T_{0}} \frac{1}{2} x^{2} \beta^{2}(s) \mathrm{d} s-i x y\right] \\
& \times\left(\mathrm{e}^{i x \psi(t, z)}-1\right) \mathrm{d} x .
\end{aligned}
$$

Next we want to find

$$
\mathbb{E}\left[D_{t} \delta_{Y}(y) \mid \mathcal{F}_{t}\right]
$$

where $D_{t}$ denotes the Hida-Malliavin derivative at $t$ with respect to Brownian motion $B$ :

Note that:

$$
\begin{aligned}
& D_{t} \delta_{Y}(y) \\
& =\frac{1}{2 \pi} \int_{\mathbb{R}} D_{t} \exp ^{\diamond}\left[\int_{0}^{T_{0}} \int_{\mathbb{R}}\left(\mathrm{e}^{i x \psi(s, \zeta)}-1\right) \tilde{N}(\mathrm{~d} s, \mathrm{~d} \zeta)+\int_{0}^{T_{0}} i x \beta(s) \mathrm{d} B(s)\right. \\
& \left.\quad+\int_{0}^{T_{0}}\left\{\int_{\mathbb{R}}\left(\mathrm{e}^{i x \psi(s, \zeta)}-1-i x \psi(s, \zeta)\right) v(\mathrm{~d} \zeta)-\frac{1}{2} x^{2} \beta^{2}(s)\right\} \mathrm{d} s-i x y\right] \mathrm{d} x
\end{aligned}
$$




$$
\begin{aligned}
= & \frac{1}{2 \pi} \int_{\mathbb{R}} \exp ^{\diamond}\left[\int_{0}^{T_{0}} \int_{\mathbb{R}}\left(\mathrm{e}^{i x \psi(s, \zeta)}-1\right) \tilde{N}(\mathrm{~d} s, \mathrm{~d} \zeta)+\int_{0}^{T_{0}} i x \beta(s) \mathrm{d} B(s)\right. \\
& \left.+\int_{0}^{T_{0}}\left\{\int_{\mathbb{R}}\left(\mathrm{e}^{i x \psi(s, \zeta)}-1-i x \psi(s, \zeta)\right) v(\mathrm{~d} \zeta)-\frac{1}{2} x^{2} \beta^{2}(s)\right\} \mathrm{d} s-i x y\right] \\
& \diamond D_{t}\left[\int_{0}^{T_{0}} \int_{\mathbb{R}}\left(\mathrm{e}^{i x \psi(s, \zeta)}-1\right) \tilde{N}(\mathrm{~d} s, \mathrm{~d} \zeta)+\int_{0}^{T_{0}} i x \beta(s) \mathrm{d} B(s)\right. \\
& \left.+\int_{0}^{T_{0}}\left\{\int_{\mathbb{R}}\left(\mathrm{e}^{i x \psi(s, \zeta)}-1-i x \psi(s, \zeta)\right) v(\mathrm{~d} \zeta)-\frac{1}{2} x^{2} \beta^{2}(s)\right\} \mathrm{d} s-i x y\right] \mathrm{d} x \\
= & \frac{1}{2 \pi} \int_{\mathbb{R}} \exp ^{\diamond}\left[\int_{0}^{T_{0}} \int_{\mathbb{R}}\left(\mathrm{e}^{i x \psi(s, \zeta)}-1\right) \tilde{N}(\mathrm{~d} s, \mathrm{~d} \zeta)+\int_{0}^{T_{0}} i x \beta(s) \mathrm{d} B(s)\right. \\
& \left.+\int_{0}^{T_{0}}\left\{\int_{\mathbb{R}}\left(\mathrm{e}^{i x \psi(s, \zeta)}-1-i x \psi(s, \zeta)\right) v(\mathrm{~d} \zeta)-\frac{1}{2} x^{2} \beta^{2}(s)\right\} \mathrm{d} s-i x y\right] i x \beta(t) \mathrm{d} x .
\end{aligned}
$$

Here we have used that

$$
D_{t} \int_{0}^{T_{0}} \int_{\mathbb{R}}\left(\mathrm{e}^{i x \psi(s, \zeta)}-1\right) \tilde{N}(\mathrm{~d} s, \mathrm{~d} \zeta)=0
$$

which follows from the assumption that $B$ and $\tilde{N}$ are independent, so for $D_{t}$ the random variable $\tilde{N}(s, \zeta)$ is like a constant.

Using Eq. (2.13) and the Wick chain rule we get

$$
\begin{aligned}
\mathbb{E}\left[D_{t} \delta_{Y}(y) \mid \mathcal{F}_{t}\right] & \\
= & \frac{1}{2 \pi} \int_{\mathbb{R}} \mathbb{E}\left[\operatorname { e x p } ^ { \diamond } \left[\int_{0}^{T_{0}} \int_{\mathbb{R}}\left(\mathrm{e}^{i x \psi(s, \zeta)}-1\right) \tilde{N}(\mathrm{~d} s, \mathrm{~d} \zeta)+\int_{0}^{T_{0}} i x \beta(s) \mathrm{d} B(s)\right.\right. \\
& +\int_{0}^{T_{0}}\left\{\int_{\mathbb{R}}\left(\mathrm{e}^{i x \psi(s, \zeta)}-1-i x \psi(s, \zeta)\right) v(\mathrm{~d} \zeta)-\frac{1}{2} x^{2} \beta^{2}(s)\right\} \mathrm{d} s \\
& \left.-i x y] i x \beta(t) \mathrm{d} x \mid \mathcal{F}_{t}\right] \mathrm{d} x \\
= & \frac{1}{2 \pi} \int_{\mathbb{R}} \mathbb{E}\left[\operatorname { e x p } ^ { \diamond } \left[\int_{0}^{T_{0}} \int_{\mathbb{R}}\left(\mathrm{e}^{i x \psi(s, \zeta)}-1\right) \tilde{N}(\mathrm{~d} s, \mathrm{~d} \zeta)+\int_{0}^{T_{0}} i x \beta(s) \mathrm{d} B(s)\right.\right. \\
& +\int_{0}^{T_{0}}\left\{\int_{\mathbb{R}}\left(\mathrm{e}^{i x \psi(s, \zeta)}-1-i x \psi(s, \zeta)\right) v(\mathrm{~d} \zeta)-\frac{1}{2} x^{2} \beta^{2}(s)\right\} \mathrm{d} s \\
& \left.-i x y] \mid \mathcal{F}_{t}\right] i x \beta(t) \mathrm{d} x \\
= & \frac{1}{2 \pi} \int_{\mathbb{R}} \exp \left[\int_{0}^{t} \int_{\mathbb{R}} i x \psi(s, \zeta) \tilde{N}(\mathrm{~d} s, \mathrm{~d} \zeta)+\int_{0}^{t} i x \beta(s) \mathrm{d} B(s)\right. \\
& \left.+\int_{t}^{T_{0}} \int_{\mathbb{R}}\left(\mathrm{e}^{i x \psi(s, \zeta)}-1-i x \psi(s, \zeta)\right) v(\mathrm{~d} \zeta) \mathrm{d} s-\int_{t}^{T_{0}} \frac{1}{2} x^{2} \beta^{2}(s) \mathrm{d} s-i x y\right] i x \beta(t) \mathrm{d} x .
\end{aligned}
$$


- (2.16) should be corrected to

$$
\|\beta\|_{[t, T]}^{2}:=\int_{t}^{T} \beta(s)^{2} \mathrm{~d} s>0 \text { for all } t \in[0, T] .
$$

- The expression after the last equality sign in (2.19) should be corrected to

$$
-\left(2 \pi\|\beta\|_{\left[t, T_{0}\right]}^{2}\right)^{-\frac{1}{2}} \exp \left[-\frac{(Y(t)-y)^{2}}{2\|\beta\|_{\left[t, T_{0}\right]}^{2}}\right] \frac{Y(t)-y}{\|\beta\|_{\left[t, T_{0}\right]}^{2}} \beta(t) .
$$

- Section 2.3 should be deleted, because the formulas (2.2)-(2.22) in Sect. 2.3 are not correct as stated. The reason is that with $\beta=0$ in (2.7) these integrals do not converge. However, the integrals do converge for all $\beta \neq 0$, and it can be proved that the limit of the quotient $\psi(t, y):=\frac{(2.12)}{(2.8)}$ with $\psi=1$ exists when $\beta \rightarrow 0$. This limit is the quantity that plays a key role in the corresponding optimal portfolio problem in Sects. 6.3 and 6.4. See (6.62) and (6.82).

- In the last line before (5.3), correct $L 2(\lambda \times P)$ to $L^{2}(\lambda \times P)$.

- In the statement of Theorem 5.1, and also in the proof, we should write $\frac{\mathrm{d}}{\mathrm{d} a} J((u+$ $a \beta)(., y))\left.\right|_{a=0}$ in stead of $\left.\frac{\mathrm{d}}{\mathrm{d} a} J(u+a \beta)\right|_{a=0}$.

- (6.40) should be corrected to

$$
\Pi^{*}(s)=\frac{b_{0}\left(s, Y\left(T_{0}\right)\right)}{\sigma_{0}^{2}\left(s, Y\left(T_{0}\right)\right)}+\frac{\left(Y\left(T_{0}\right)-Y(s)\right) \beta(s)}{\sigma_{0}\left(s, Y\left(T_{0}\right)\right)\|\beta\|_{\left[s, T_{0}\right]}^{2}}, \quad 0 \leq s \leq T<T_{0} .
$$

- From (6.107) to (6.109) we should write:

Theorem 6.9

Suppose $Y$ is as in (2.5). Then the processes $\phi(t, y)$ and $\psi(t, y, z)$ in the Eq. (6.106) for the optimal portfolio $\pi(t, y)$ have the following expressions:

$$
\begin{aligned}
\phi(t, y) & =\frac{i \beta(t) \int_{\mathbb{R}} F(t, x, y) x \mathrm{~d} x}{\int_{\mathbb{R}} F(t, x, y) \mathrm{d} x} \\
\psi(t, y, z) & =\frac{\int_{\mathbb{R}} F(t, x, y)\left(\mathrm{e}^{i x \psi(t, z)}-1\right) \mathrm{d} x}{\int_{\mathbb{R}} F(t, x, y) \mathrm{d} x}
\end{aligned}
$$

where

$$
\begin{aligned}
F(t, x, y)= & \int_{\mathbb{R}} \exp \left[\int_{0}^{t} \int_{\mathbb{R}} i x \psi(s, \zeta) \tilde{N}(\mathrm{~d} s, \mathrm{~d} \zeta)+\int_{0}^{t} i x \beta(s) \mathrm{d} B(s)\right. \\
& +\int_{t}^{T_{0}} \int_{\mathbb{R}}\left(\mathrm{e}^{i x \psi(s, \zeta)}-1-i x \psi(s, \zeta)\right) v(\mathrm{~d} \zeta) \mathrm{d} s \\
& \left.-\int_{t}^{T_{0}} \frac{1}{2} x^{2} \beta^{2}(s) \mathrm{d} s-i x y\right] \mathrm{d} x .
\end{aligned}
$$

